\title{
Relationship between small and large strain solutions for general cavity expansion problems in elasto-plastic soils
}

\author{
Apostolos Vrakas \\ Institute for Geotechnical Engineering, ETH Zurich, Switzerland \\ Stefano-Franscini-Platz 5, 8093 Zurich, Switzerland \\ Tel.: +414463304 68; Fax: +41446331097 \\ E-Mail address: apostolos.vrakas@igt.baug.ethz.ch
}

\begin{abstract}
This paper presents a closed-form relationship between small and finite strain cavity expansion solutions, based on the non-linearly elastic-perfectly plastic cylindrical (or spherical) problem with a general Mohr's criterion and constant plastic dilatancy. It is shown, however, that this relationship is sufficiently accurate for general expansion problems not obeying plane-strain rotationally (or spherically) symmetric conditions and involving strainhardening/softening constitutive behaviour. Therefore, it quantifies the error stemming from the computational assumption of small deformations and provides a simple and efficient way of accounting for geometric non-linearity based entirely on conventional small strain computational methods: 'self-correction' of small strain analyses results.
\end{abstract}

Keywords: cavity expansion; deformation; hyperbolic function; plasticity

\section{Introduction}

Cavity expansion theory has been widely studied in the literature. A summary of numerous analytical solutions as well as of the relevant geotechnical applications has been presented by $\mathrm{Yu}$ [1] and will not be repeated here. The present paper focuses on the influence of the deformation formulation considered in the analysis of general cavity expansion problems.

It is well-known that solutions based on small strain theory predict a continuously increasing cavity pressure (see for example the dashed curve in Fig. 1 for the idealised case of isotropic cylindrical cavity expansion in a Mohr-Coulomb material), while cavity expansion solutions based on finite strain theory predict a limit value at large expansions (see for example the solid curve in Fig. 1). Small strain theory therefore leads to predictions of stiffer system behaviour as it is unable to reproduce an ultimate state (e.g. Baguelin et al. [2]; Carter et al. [3]; Soulié et al. [4]; Yu \& Houlsby [5]).

This paper presents a novel closed-form relationship between small and finite strain elastoplastic cavity expansion solutions (Section 2). The derivation of this relationship is based on several simplifying assumptions (Section 3), but it is shown by means of modelling an elliptical and an ellipsoidal cavity as well as a finite length self-boring pressuremeter test in 
modified Cam-clay soils under drained and undrained conditions that it is sufficiently accurate for general elasto-plastic expansion problems not obeying plane strain axial (or spherical) symmetry, with rotating principal directions (Section 4).

\section{The relationship between small and large strain solutions}

It will be proven in the following that the normalized wall displacement $U_{a, l s}\left(=u_{a, l s} / a_{0}\right.$, where $a_{0}$ denotes the initial cavity radius; Fig. 2a) obtained from a large strain analysis of cavity expansion in elasto-plastic soils satisfying a general Mohr's failure criterion and exhibiting constant plastic dilatancy can be expressed with respect to the normalized wall displacement $U_{a, s s}\left(=u_{a, s s} / a_{0}\right.$; Fig. $\left.2 \mathrm{~b}\right)$ obtained from a small strain analysis (and corresponding to the same cavity pressure) through the following hyperbolic function:

$$
U_{a, l s}=h\left(U_{a, s s}\right)=\frac{1}{\left[1-\left(\zeta / \kappa_{\psi}+1\right) U_{a, s s}\right]^{\frac{1}{\zeta / \kappa_{\psi}+1}}}-1,
$$

where the variable $\zeta$ indicates the cavity type $\left(\zeta=1\right.$, cylindrical; $\zeta=2$, spherical) and $\kappa_{\psi}$ is the dilation constant, expressed in terms of the dilation angle $\psi$ as $\kappa_{\psi}=(1+\sin \psi) /(1-\sin \psi)$. The large strain wall displacement tends to infinity as the small strain solution approaches the critical value

$$
U_{a, \lim }=\frac{1}{\zeta / \kappa_{\psi}+1}
$$

which implies that the limit pressure $\sigma_{a, \lim }$, that is, the pressure required to create a cavity or to enlarge a cavity of finite radius indefinitely, corresponds to the cavity pressure obtained by means of a small strain analysis at $U_{a, \lim }$ (see for example the circular markers in Fig. 1). Setting $\kappa_{\psi} \rightarrow \infty$, which corresponds to the limit case of an infinitely dilatant material $(\psi=$ $90^{\circ}$ ), leads to the simplified relationship

$$
U_{a, l s}=h\left(U_{a, s s}\right)=\frac{U_{a, s s}}{1-U_{a, s s}} .
$$

Figures 3 and 4 plot Eq. (1) for cylindrical and spherical cavity expansion, respectively, considering several dilation angles. The identity line is included in order to illustrate the small strain solution error, which, as can be seen, is practically negligible up to expansions of $10 \%$, but increases significantly for greater values.

The hyperbolic function $h$ satisfies all of the required conditions for theoretical consistency: (i) $h(0)=0$ and $h^{\prime}(0)=1$ (i.e. coincides with the small strain solution as $U_{a, s s}$ tends to zero); (ii) $h\left(U_{a, \lim }\right)=h^{\prime}\left(U_{a, \lim }\right)=\infty$ (i.e. approaches infinity asymptotically as $U_{a, s s}$ tends to $\left.U_{a, \mathrm{lim}}\right)$; (iii) $h^{\prime}\left(U_{a, s s}\right)>0$ and $h^{\prime \prime}\left(U_{a, s s}\right)>0$ (i.e. is a monotone increasing and convex function). 


\section{Derivation of Equation (1)}

\subsection{Problem statement}

The proof of Eq. (1) follows the same line as that presented by Vrakas \& Anagnostou [6] for the tunnel excavation problem and is based on the following assumptions:

(i) A cylindrical $(\zeta=1)$ or spherical $(\zeta=2)$ cavity of radius $a_{0}$ is embedded in an infinite, homogeneous, isotropic and weightless medium (Fig. 2).

(ii) The initial stress field is uniform and isotropic of magnitude $\sigma_{0}$ (for the cylindrical cavity, however, the initial out-of-plane stress should not necessarily equal the in-plane stresses).

(iii) The cavity pressure is increased from $\sigma_{0}$ to $\sigma_{a}$, while the stresses in the far field remain unaltered by the cavity expansion.

(iv) The ground is assumed to be (non-linearly) elastic - perfectly plastic, satisfying a general (non-linear) Mohr's criterion that does not depend on the intermediate principal stress and exhibiting constant plastic dilatancy.

(v) The out-of-plane direction remains the intermediate principal direction throughout elastoplastic loading in the plane strain problem.

(vi) The strains in the elastic zone are small.

(vii) The elastic strains are disregarded in the plastic zone, i.e. the elastic strains are considerably smaller than the plastic strains.

(viii) 'Total stresses' are used, considering the influence of water to be negligible, which is the case for dry or completely drained conditions.

Although these simplifying assumptions may seem restrictive, exact computations that disregard some or all of them still verify the derived relationship between small and large strain cavity expansion solutions, as will be shown in the following.

Compressive normal stresses, $\sigma$, and strains, $\varepsilon$, as well as outward radial displacements, $u$, are taken as positive. The analysis is performed in cylindrical and spherical polar coordinates, respectively, and the subscripts ' $s s$ ' and ' $l s$ ' are used to differentiate between the small and large strain formulation variables. According to the large strain formulation, a clear distinction is made between the undeformed and the deformed configuration of the continuum, taking the material, $r_{0}$, and the spatial, $r$, radial coordinates of each material point, in contrast to the infinitesimal strain formulation, which assumes that deformations are small, such that $r_{0} \approx r$. It should be noted, finally, that, due to the symmetry of the problem, the state of each material point is on the same stress and strain path (e.g. Hill [7], Chadwick [8], Yu \& Carter [9]), which allows the following theoretical analysis.

\subsection{Considerations for the elasto-plastic boundary}

The ground response is elastic until the yield condition is satisfied at the cavity wall. As the cavity pressure increases, the ground around the cavity yields, forming an expanding plastic 
ring of outer radius $\rho$ (Fig. 2). The elastic zone then constitutes an infinite ring of inner radius $\rho$, which is at the onset of yielding, with the elastically computed radial and tangential stresses at $r=\rho$ satisfying the Mohr's criterion. As the elastic strains are small, there is no difference between the large and small deformation formulation with respect to stresses and strains in the elastic zone (even though the plastic zone radii are different), and thus,

$$
\sigma_{\rho, l s} \cong \sigma_{\rho, s s}
$$

and

$$
\varepsilon_{t \rho, l s} \cong \varepsilon_{t \rho, s s},
$$

where $\sigma_{\rho}$ and $\varepsilon_{t \rho}$ denote the radial stress and tangential strain at the elasto-plastic boundary, respectively.

\subsection{Kinematic considerations in the plastic zone}

The plastic flow rule implies that the ratio of incremental plastic strains is constant:

$$
\frac{\mathrm{d} \varepsilon_{r}^{p l}}{\mathrm{~d} \varepsilon_{t}^{p l}}=-\frac{\zeta}{\kappa_{\psi}} .
$$

Integrating Eq. (6) over the elasto-plastic deformation history of a material point and recalling that elastic strains are negligible inside the plastic zone relative to plastic strains gives (Hughes et al. [10]; Palmer \& Mitchell [11]; Bigoni \& Laudiero [12])

$$
\frac{\varepsilon_{r}}{\varepsilon_{t}} \cong \frac{\varepsilon_{r}^{p l}}{\varepsilon_{t}^{p l}}=-\frac{\zeta}{\kappa_{\psi}} .
$$

In the small deformation analysis, the kinematic relationships read as follows:

$$
\varepsilon_{r, s s}=-\frac{\mathrm{d} u_{s s}}{\mathrm{~d} r}, \quad \varepsilon_{t, s s}=-\frac{u_{s s}}{r}
$$

Substituting Eq. (8) into Eq. (7) leads to a differential equation for radial displacement, the integration of which provides the following relationship between the displacements at the cavity wall, $u_{a}$, and at the elasto-plastic interface, $u_{\rho}$ :

$$
u_{a, s s} a_{0}^{\zeta / \kappa_{\psi}}=u_{\rho, s s} \rho_{s s}^{\zeta / \kappa_{\psi}}
$$

Eq. (9) expresses the incompressibility condition for $\kappa_{\psi}=1$ (Hill [7]). The tangential strain at the elasto-plastic boundary reads

$$
\varepsilon_{t \rho, s s}=-\frac{u_{\rho, s s}}{\rho_{s s}}
$$

and thus Eq. (9) can be written as 


$$
U_{a, s s}=-\varepsilon_{t \rho, s s}\left(\frac{\rho_{s s}}{a_{0}}\right)^{\zeta / \kappa_{\psi}+1} .
$$

In the large deformation analysis, logarithmic strains are used (Chadwick [8]):

$$
\varepsilon_{r, l s}=\ln \frac{\mathrm{d} r_{0}}{\mathrm{~d} r}, \quad \varepsilon_{t, l s}=\ln \frac{r_{0}}{r} .
$$

By substituting Eq. (12) into Eq. (7) (which implies that large strains are due entirely to plasticity) another differential equation is obtained, whose integration over the plastic zone leads to the following relationship:

$$
a^{\zeta / \kappa_{\psi}+1}-a_{0}^{\zeta / \kappa_{\psi}+1}=\rho_{l s}^{\zeta / \kappa_{\psi}+1}-\rho_{0, l s}^{\zeta / \kappa_{\psi}+1}
$$

where $a$ and $\rho_{0, l s}$ denote the current cavity radius, and the plastic zone radius in the undeformed configuration, respectively (Fig. 2a). Eq. (13) expresses the incompressibility condition for $\kappa_{\psi}=1$, which in the finite deformation analysis means that the two shaded areas in Fig. 2a are equal (Hill [7]). By definition,

$$
\rho_{0, l s}=\rho_{l s}-u_{\rho, l s}
$$

and thus Eq. (13) can be written as

$$
a^{\zeta / \kappa_{\psi}+1}-a_{0}^{\zeta / \kappa_{\psi}+1}=\left[1-\left(1-\frac{u_{\rho, l s}}{\rho_{l s}}\right)^{\zeta / \kappa_{\psi}+1}\right] \rho_{l s}^{\zeta / \kappa_{\psi}+1} .
$$

Neglecting second and higher order terms in both the Taylor series of the tangential strain at the elasto-plastic boundary, i.e.

$$
\varepsilon_{t \rho, l s}=\ln \left(1-\frac{u_{\rho, l s}}{\rho_{l s}}\right) \cong-\frac{u_{\rho, l s}}{\rho_{l s}},
$$

and in the resulting binomial series in Eq. (15) (since strains in the elastic zone are small), i.e.

$$
\left(1+\varepsilon_{t \rho, l s}\right)^{\zeta / \kappa_{\psi}+1} \cong 1+\left(\zeta / \kappa_{\psi}+1\right) \varepsilon_{t \rho, l s},
$$

Eq. (15) becomes

$$
a^{\zeta / \kappa_{\psi}+1}-a_{0}^{\zeta / \kappa_{\psi}+1}=-\left(\zeta / \kappa_{\psi}+1\right) \varepsilon_{t \rho, l s} \rho_{l s}^{\zeta / \kappa_{\psi}+1} .
$$

Taking further into account that (by definition)

$$
a=a_{0}+u_{a, l s},
$$

Eq. (18) leads to 


$$
U_{a, l s}=\frac{1}{\left[1+\left(\zeta / \kappa_{\psi}+1\right) \varepsilon_{t \rho, l s}\left(\frac{\rho_{l s}}{a}\right)^{\zeta / \kappa_{\psi}+1}\right]^{\frac{1}{\zeta / \kappa_{\psi}+1}}}-1
$$

Eqs. (20), (11) and (5) show that, in order to establish a relationship between the small and the large strain cavity expansion solutions, we have to relate the corresponding normalized plastic zone radii. This will be done in the following section.

\subsection{Static considerations in the plastic zone}

The stress field in the plastic zone satisfies both the yield criterion, according to which the minor (tangential) principal Cauchy stress is expressed as an arbitrary function of the major (radial) principal Cauchy stress:

$$
\sigma_{t}=f\left(\sigma_{r}\right)
$$

and the equilibrium condition, namely

$$
\frac{\mathrm{d} \sigma_{r}}{\mathrm{~d} r}+\zeta \frac{\sigma_{r}-\sigma_{t}}{r}=0
$$

These equations lead to the following differential equation for the radial stress:

$$
\frac{\mathrm{d} r}{r}=-\frac{1}{\zeta} \frac{\mathrm{d} \sigma_{r}}{\sigma_{r}-f\left(\sigma_{r}\right)}
$$

whose integration over the plastic zone, i.e. over $\left[a_{0}, \rho_{s s}\right]$ for the small strain formulation (Fig. 2b) and over $\left[a, \rho_{l s}\right]$ for the large strain formulation (Fig. 2a), yields

and

$$
\begin{aligned}
& \ln \frac{\rho_{s s}}{a_{0}}=-\frac{1}{\zeta} \int_{a_{0}}^{\rho_{s s}} \frac{\mathrm{d} \sigma_{r}}{\sigma_{r}-f\left(\sigma_{r}\right)}=F\left(\sigma_{a}\right)-F\left(\sigma_{\rho, s s}\right) \\
& \ln \frac{\rho_{l s}}{a}=-\frac{1}{\zeta} \int_{a}^{\rho_{l s}} \frac{\mathrm{d} \sigma_{r}}{\sigma_{r}-f\left(\sigma_{r}\right)}=F\left(\sigma_{a}\right)-F\left(\sigma_{\rho, l s}\right),
\end{aligned}
$$

respectively, where $F$ stands for the indefinite integral of the function $1 /$ [ $\left.\zeta\left(\sigma_{r}-f\left(\sigma_{r}\right)\right)\right]$. Eq. (4) suggests that the right hand sides of Eqs. (24) and (25) are equal and hence,

$$
\frac{\rho_{l s}}{a}=\frac{\rho_{s s}}{a_{0}}
$$

\subsection{Synthesis of the above theoretical considerations}

The combination of Eqs. (20), (11), (5) and (26) leads to Eq. (1), according to which the wall displacement from large strain analysis can be expressed as a hyperbolic function of the wall displacement from small strain analysis, for any given cavity pressure $\sigma_{a}$. Furthermore, the ratio of plastic zone radii equals (after Eqs. 26 and 1) 


$$
\frac{\rho_{l s}}{\rho_{s s}}=1+U_{a, l s}=\frac{1}{\left[1-\left(\zeta / \kappa_{\psi}+1\right) U_{a, s s}\right]^{\frac{1}{\zeta / \kappa_{\psi}+1}}},
$$

which shows that the extent of yielding is also underestimated by small strain theory (at a given $\sigma_{a}$ ).

\subsection{Discussion}

The main assumption facilitating the derivation of Eq. (1) is that of disregarding elastic strains in the plastic zone (for both deformation formulations; assumption (vii)). In fact, this assumption can influence the predicted results significantly (e.g. Carter et al. [13]; Bigoni \& Laudiero [12]; Yu \& Houlsby [5]) and indicates here that the derived hyperbolic function relates approximate large strain to approximate small strain elasto-plastic cavity expansion solutions, being exact only in the special case of incompressible and non-dilatant $\left(\kappa_{\psi}=1\right)$ ground response where the above kinematic considerations (Eqs. 9 and 13) are rigorous. The latter is the case when the undrained behaviour of saturated ground is examined. Extensive parametric studies have shown, however, that although this assumption was necessary for proving Eq. (1), it is not essential with regard to accuracy, i.e. Eq. (1) relates exact small strain to exact large strain solutions. For example, in the simplest case of the linearly elastic perfectly plastic Mohr-Coulomb model, the accuracy of Eq. (1) can directly be shown using the exact closed-form solutions of Yu \& Houlsby [5] ( $c f$. Fig. 1). For other more complicated constitutive models of dilatant cohesive-frictional grounds (note that Eq. 1 is not restricted to linear elasticity and to linear failure criteria - see assumption (iv)), the accuracy of Eq. (1) can be shown through one-dimensional semi-analytical or numerical computations.

Another basic assumption facilitating the derivation of Eq. (1) is that of perfectly plastic behaviour (assumption $(i v)$ ). It can be proved, however, considering that failure conditions are reached after a certain amount of shearing, that Eq. (1) (with $\kappa_{\psi}=1$ ) is accurate also for cavity expansions in critical state soils (or hardening/softening in general; e.g. Papanastasiou \& Durban [14]). More specifically, at higher cavity pressures, the plastic zone consists of an inner critical state (or perfectly plastic) ring and an outer hardening/softening plastic ring. Considering that hardening/softening ends at strains lower than $10 \%$, the difference between the small and finite strain solutions with respect to the outer plastic zone is negligible and the stresses and strains at the interface between the two plastic rings are practically the same, despite that the position of the interface is different. Therefore, the above kinematic (with $\kappa_{\psi}=$ 1 , implying zero plastic volumetric deformations) and static considerations also hold for a critical state (or hardening/softening in general) material, with $\rho$ corresponding to the outer radius of the inner critical state (or perfectly plastic) ring. For instance, during drained cavity expansion in critical state soils, the stress state of each material point approaches the critical state asymptotically, with the corresponding stress path moving practically on the critical state line (Collins \& Stimpson [15]; Chen \& Abousleiman [16]). On the other hand, under undrained loading conditions, the effective stresses and thus the deviatoric stress $\sigma_{r}-\sigma_{t}(c f$. Eq. 22) around the cavity wall remain constant after certain expansion (Randolph et al. [17]; Collins \& Yu [18]; Cao et al. [19]; Chen \& Abousleiman [20]; Li et al. [21]). 


\section{Validity of Equation (1) for general expansion problems}

This section examines the validity of Eq. (1) for cavity expansion problems not obeying plane strain rotationally symmetric or spherically symmetric conditions. All the numerical computations were performed with the Abaqus finite element code (Dassault Systèmes [22]). The Mohr-Coulomb analyses (Section 4.1) were performed using 4-node quadrilateral plane strain or axisymmetric elements and the stress point algorithm after Clausen et al. [23], while the modified Cam-clay analyses (Section 4.2) were performed using 8-node quadrilateral axisymmetric elements and the Abaqus built-in subroutine. Furthermore, the finite deformation analyses were based on an updated Lagrangian rate-type plasticity formulation (i.e. the constitutive equations relate the Jaumann rate of the Cauchy stress tensor to the rate of deformation tensor, which is decomposed into elastic and plastic parts).

\subsection{Elliptical and ellipsoidal cavity expansions}

The elliptical (e.g. Zhou et al. [24]) and ellipsoidal (e.g. McMahon et al. [25]) cavity expansions in a Mohr-Coulomb material are examined first. The ratio of the major-axis radius, $b_{1}$, to the minor-axis radius, $b_{2}$, is set equal to 2 . The initial stress field is taken as isotropic, with the tractions at the remote boundaries (placed $200 b_{1}$ from the centre of the cavity) remaining fixed during the successive increase of the cavity pressure. Figures 5 and 6 show the applied pressure as a function of the computed average normalized cavity wall displacement for the plane strain (elliptical) and the axisymmetric (ellipsoidal) problem, respectively. As can be seen, the small strain analyses (dashed curves) lead to much stiffer behaviour compared with the finite strain analyses (solid curves) for deformations greater than $10 \%$, while application of Eq. (1) (circular markers) provides highly accurate results. It can be shown in a similar way that Eq. (1) is valid also for displacement-controlled expansions under a non-uniform pressure (e.g. Zhou et al. [26]).

\subsection{Finite length self-boring pressuremeter test}

The second example considers a self-boring pressuremeter with length to diameter ratio of 6 . It should be emphasized that intention here is not to study the effect of pressuremeter geometry on the interpretation of test results (for such investigations see Houlsby \& Carter [27], Shuttle \& Jefferies [28], Yu et al. [29]), but to verify the accuracy of the derived relationship in a typical cavity expansion problem with rotating principal directions. Therefore, a simple numerical model of the finite length self-boring pressuremeter is used (Fig. 7). The depth of penetration as well as the extent of the axisymmetric model in the horizontal direction is taken sufficiently high, while the body of the pressuremeter is considered as rigid. Constant stress boundary conditions are imposed at the far boundaries, while a successively increasing (from the in situ horizontal stress) uniform pressure is applied at the elements adjacent to the pressuremeter membrane. The ground is assumed to satisfy the two-invariant modified Cam-clay model (Roscoe \& Burland [30]). Four parameter sets are analysed (normally consolidated and heavily over-consolidated London and Boston Blue clay, respectively) under both drained (without water) and undrained conditions (note that Fig. 7 is plotted for zero initial pore pressure). Obviously, all the simplifying assumptions facilitating the derivation of Eq. (1) are violated here. 
Figure 8a shows the computed small strain (dashed) and finite strain (solid) pressureexpansion curves and the corresponding curves obtained by correcting the small strain analyses results according to Eq. (1) (with $\zeta=\kappa_{\psi}=1$; circular markers) or Eq. (3) (square markers) for a normally consolidated $(O C R=1.01)$ and a heavily over-consolidated $(O C R=$ 10) London clay under fully drained conditions, while Figure $8 b$ shows the corresponding undrained results. Figure 9 refers to Boston Blue clay. The present results verify the sufficiency of the derived hyperbolic function: Eqs. (1) and (3) bound the pressure-expansion curves obtained from rigorous finite strain numerical analyses, slightly underestimating or overestimating, respectively, the applied pressure at a given cavity expansion.

\section{Conclusion}

The paper presented a closed-form relationship between small and finite strain elasto-plastic cavity expansion solutions (Eq. 1). Although the proof of this relationship requires certain simplifying assumptions, these assumptions are not essential with regard to its overall validity. More specifically, it was shown that the derived relationship is sufficiently accurate for general elasto-plastic expansion problems not satisfying plane strain axial (or spherical) symmetry, and involving strain hardening/softening constitutive behaviour as well as drained or undrained loading conditions. Therefore, the error stemming from the computational assumption of small deformations was quantified and a direct way for taking account of geometric non-linearity based entirely on conventional small strain computational methods was proposed. Of course, Eq. (1) applies to monotonic/static cavity expansions; rigorous finite deformation analyses are indispensable for more complex cases (cf. Wang et al. [31]).

\section{Acknowledgements}

This paper evolved within the framework of the research project 'Analysis of large deformation problems in tunnelling considering geometric nonlinearities', which is being performed at the ETH Zurich with the financing of the Swiss National Science Foundation (SNF) under Project No. 200021_153433. I wish to thank Prof. Dr. G. Anagnostou (ETH Zurich) for his guidance, encouragement and support during preparation of the manuscript. 


\section{Notation}

\begin{tabular}{|c|c|}
\hline$a, a_{0}$ & current and initial cavity radius \\
\hline$b_{1}, b_{2}$ & major- and minor-axis radius of an ellipse/ellipsoid \\
\hline$c$ & cohesion \\
\hline E & Young's modulus \\
\hline$F$ & indefinite integral of the function $1 /\left[\zeta\left(\sigma_{r}-f\left(\sigma_{r}\right)\right)\right]$ \\
\hline$f$ & arbitrary function of the major principal stress \\
\hline$h$ & hyperbolic function \\
\hline$K_{0}$ & earth pressure coefficient at rest \\
\hline$M$ & slope of the critical state line \\
\hline$O C R$ & one-dimensional over-consolidation ratio \\
\hline$R$ & isotropic over-consolidation ratio \\
\hline$r, r_{0}$ & current and initial radius of a material point \\
\hline$U_{a}$ & normalized cavity wall displacement $\left(=u_{a} / a_{0}\right)$ \\
\hline$U_{a, \lim }$ & critical (small strain) normalized wall displacement \\
\hline$u$ & radial displacement of a material point \\
\hline$u_{1}, u_{2}$ & radial displacement at the major and at the minor axis of an ellipse/ellipsoid \\
\hline$u_{a}$ & radial displacement at the cavity wall \\
\hline$u_{\rho}$ & radial displacement at the elasto-plastic boundary \\
\hline$\varepsilon_{r}, \varepsilon_{t}$ & radial and tangential strain \\
\hline$\varepsilon_{r}^{p l}, \varepsilon_{t}^{p l}$ & radial and tangential plastic strain \\
\hline$\varepsilon_{t \rho}$ & tangential strain at the elasto-plastic boundary \\
\hline$\zeta$ & variable indicating the type of cavity $(\zeta=1$, cylindrical; $\zeta=2$, spherical $)$ \\
\hline$\kappa_{\psi}$ & function of dilation angle \\
\hline$\kappa, \lambda$ & $\begin{array}{l}\text { slope of the swelling and of the normal consolidation line in the semi- } \\
\text { logarithmic compression plane }\end{array}$ \\
\hline$v$ & Poisson's ratio \\
\hline$\rho, \rho_{0}$ & radius of the plastic zone in the current and in the undeformed state \\
\hline$\sigma_{0}, \sigma_{0}^{\prime}$ & initial isotropic total and effective stress \\
\hline$\sigma_{h 0}, \sigma_{v 0}$ & initial horizontal and vertical stress \\
\hline$\sigma_{a}$ & cavity pressure \\
\hline$\sigma_{a, \lim }$ & limit cavity pressure \\
\hline$\sigma_{r}, \sigma_{t}$ & radial and tangential Cauchy stress \\
\hline$\sigma_{\rho}$ & radial stress at the elasto-plastic boundary \\
\hline$v_{0}$ & initial specific volume \\
\hline$\varphi, \psi$ & friction and dilation angle \\
\hline
\end{tabular}

Subscripts 


\section{References}

[1] Yu HS. Cavity expansion methods in geomechanics. Dordrecht: Springer; 2000.

[2] Baguelin F, Jézéquel JF, Shields DH. The pressuremeter and foundation engineering. Clausthal: Trans Tech Publications; 1978.

[3] Carter JP, Randolph MF, Wroth CP. Stress and pore pressure changes in clay during and after the expansion of a cylindrical cavity. Int J Numer Anal Meth Geomech 1979; 3(4): 305322.

[4] Soulié M, Ladanyi B, Degenne P. Expansion of a cylindrical cavity in a very deformable medium: a theoretical study. In: Biraud J-L, Audibert JME, editors. The Pressuremeter and its Marine Applications: Second International Symposium, ASTM STP 950, ASTM: Philadelphia; 1986, p. 232-244.

[5] Yu HS, Houlsby GT. Finite cavity expansion in dilatant soils: loading analysis. Géotechnique 1991; 41(2): 173-183.

[6] Vrakas A, Anagnostou G. A simple equation for obtaining finite strain solutions from small strain analyses of tunnels with very large convergences. Géotechnique 2015; 65(11): 936-944.

[7] Hill R. The mathematical theory of plasticity. London: Oxford University Press; 1950.

[8] Chadwick P. The quasi-static expansion of a spherical cavity in metals and ideal soils. Quarterly Journal of Mechanics and Applied Mathematics 1959; 12(1): 52-71.

[9] Yu HS, Carter JP. Rigorous similarity solutions for cavity expansion in cohesive-frictional soils. International Journal of Geomechanics 2002; 2(2): 233-258.

[10] Hughes JMO, Wroth CP, Windle D. Pressuremeter tests in sands. Géotechnique 1977; 27(4): 455-477.

[11] Palmer AC, Mitchell RJ. Plane-strain expansion of a cylindrical cavity in clay. In: Parry RHG, editor. Stress-Strain Behaviour of Soils: Proceedings of the Roscoe Memorial Symposium, Henley-on-Thames: Foulis; 1972, p. 588-599.

[12] Bigoni D, Laudiero F. The quasi-static finite cavity expansion in a non-standard elastoplastic medium. International Journal of Mechanical Sciences 1989; 31(11): 825-837.

[13] Carter JP, Booker JR, Yeung SK. Cavity expansion in cohesive frictional soils. Géotechnique 1986; 36(3): 349-358.

[14] Papanastasiou P, Durban D. Elastoplastic analysis of cylindrical cavity problems in geomaterials. Int J Numer Anal Meth Geomech 1997; 21(2): 133-149.

[15] Collins IF, Stimpson JR. Similarity solutions for drained and undrained cavity expansions in soils. Géotechnique 1994; 44(1): 21-34. 
[16] Chen SL, Abousleiman YN. Exact drained solution for cylindrical cavity expansion in modified Cam Clay soil. Géotechnique 2013; 63(6): 510-517.

[17] Randolph MF, Carter JP, Wroth CP. Driven piles in clay - the effects of installation and subsequent consolidation. Géotechnique 1979; 29(4): 361-393.

[18] Collins IF, Yu HS. Undrained cavity expansions in critical state soils. Int J Numer Anal Meth Geomech 1996; 20(7): 489-516.

[19] Cao LF, Teh CI, Chang MF. Undrained cavity expansion in modified Cam clay I: Theoretical analysis. Géotechnique 2001; 51(4): 323-334.

[20] Chen SL, Abousleiman YN. Exact undrained elasto-plastic solution for cylindrical cavity expansion in modified Cam Clay soil. Géotechnique 2012; 62(5): 447-456.

[21] Li L, Li J, Sun D. Anisotropically elasto-plastic solution to undrained cylindrical cavity expansion in $K_{0}$-consolidated clay. Computers and Gotechnics 2016; 73: 83-90.

[22] Dassault Systèmes. Abaqus 6.12 - Theory Manual and Analysis User's Manual; 2012.

[23] Clausen J, Damkilde L, Andersen, L. An efficient return algorithm for non-associated plasticity with linear yield criteria in principal stress space. Computers and Structures 2007; 85(23-24): 1795-1807.

[24] Zhou H, Liu H, Kong G, Cao Z. Analytical solution for pressure-controlled elliptical cavity expansion in elastic-perfectly plastic soil. Géotechnique Letters 2014; 4(2): 72-78.

[25] McMahon BT, Haigh SK, Bolton MD. Cavity expansion model for the bearing capacity and settlement of circular shallow foundations on clay. Géotechnique 2013; 63(9): 746-752.

[26] Zhou H, Liu H, Kong G, Huang X. Analytical solution of undrained cylindrical cavity expansion in saturated soil under anisotropic initial stress. Computers and Geotechnics 2014; 55: 232-239.

[27] Houlsby GT, Carter JP. The effects of pressuremeter geometry on the results of tests in clay. Géotechnique 1993; 43(4): 567-576.

[28] Shuttle DA, Jefferies MG. A practical geometry correction for interpreting pressuremeter tests in clay. Géotechnique 1995; 45(3): 549-553.

[29] Yu HS, Charles MT, Khong CD. Analysis of pressuremeter geometry effects in clay using critical state models. Int J Numer Anal Meth Geomech 2005; 29(8): 845-859.

[30] Roscoe KH, Burland JB. On the generalized stress-strain behaviour of 'wet' clay. In: Heyman J, Leckie FA, editors. Engineering Plasticity, Cambridge: Cambridge University Press; 1968, p. 535-609.

[31] Wang D, Bienen B, Nazem M, Tian J, Zheng Y, Pucker T, Randolph M. Large deformation finite element analyses in geotechnical engineering. Computers and Geotechnics 2015; 65: 104-114. 


\section{Figure captions}

Figure 1. Small strain, large strain and corrected small strain (Eq. 1) cylindrical cavity expansion curves based on the Mohr-Coulomb yield criterion

Figure 2. Setup of the cylindrical (or spherical) cavity excavation problem for, (a), large strain formulation and, (b), small strain formulation

Figure 3. Normalized cavity wall displacement from large strain analysis as a function of the normalized cavity wall displacement from small strain analysis of cylindrical cavity expansion, considering several dilation angles (Eq. 1)

Figure 4. Normalized cavity wall displacement from large strain analysis as a function of the normalized cavity wall displacement from small strain analysis of spherical cavity expansion, considering several dilation angles (Eq. 1)

Figure 5. Normalized applied pressure as a function of the average normalized wall displacement of an elliptical cavity based on the Mohr-Coulomb model

Figure 6. Normalized applied pressure as a function of the average normalized wall displacement of an ellipsoidal cavity based on the Mohr-Coulomb model

Figure 7. The axisymmetric numerical model of the finite length self-boring pressuremeter

Figure 8. Normalized applied pressure as a function of the normalized wall displacement at the centre of the pressuremeter membrane during expansion in normally consolidated $(O C R=$ $\left.1.01-K_{0}=0.62, R=1.36\right)$ and heavily over-consolidated $\left(O C R=10-K_{0}=1.49, R=7.53\right)$ London clay under (a) fully drained and (b) undrained conditions $(v=0.3, \kappa=0.062, \lambda=$ $0.161, M=0.888, v_{0}=2$ )

Figure 9. Normalized applied pressure as a function of the normalized wall displacement at the centre of the pressuremeter membrane during expansion in normally consolidated ( $O C R=$ $\left.1.01-K_{0}=0.50, R=1.40\right)$ and heavily over-consolidated $\left(O C R=10-K_{0}=1.58, R=6.67\right)$ Boston Blue clay under (a) fully drained and (b) undrained conditions $(v=0.3, \kappa=0.03, \lambda=$ $0.15, M=1.2, v_{0}=2$ ) 

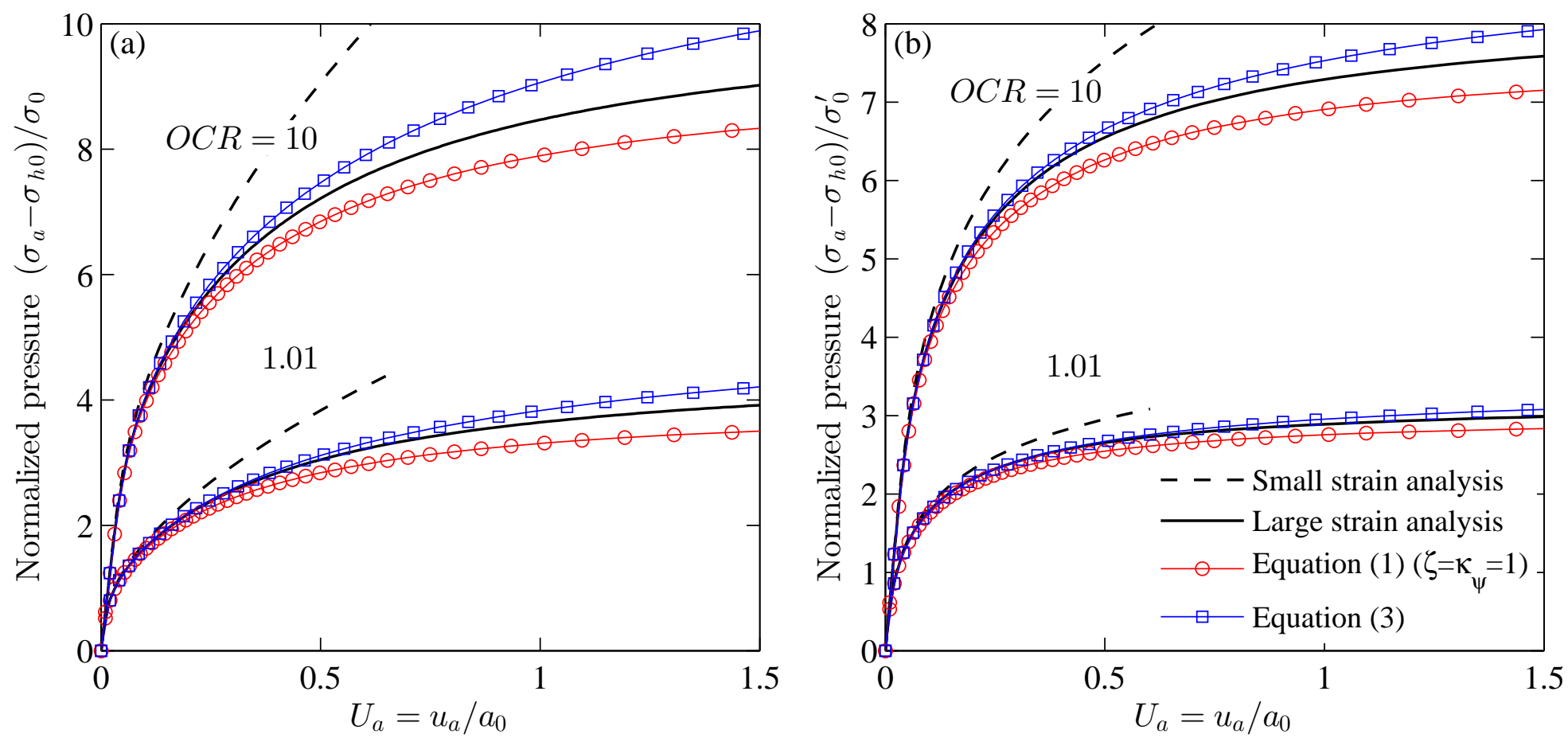

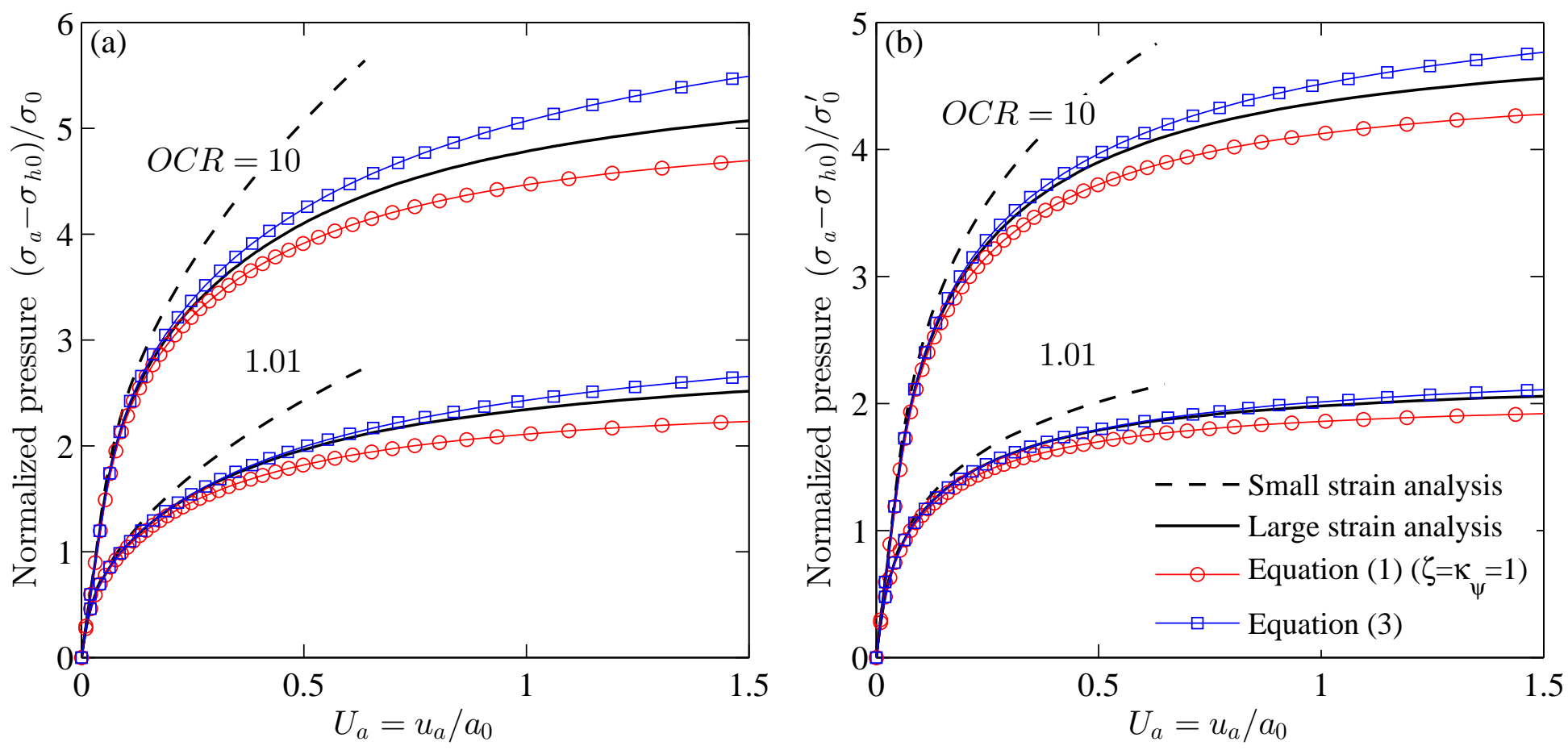


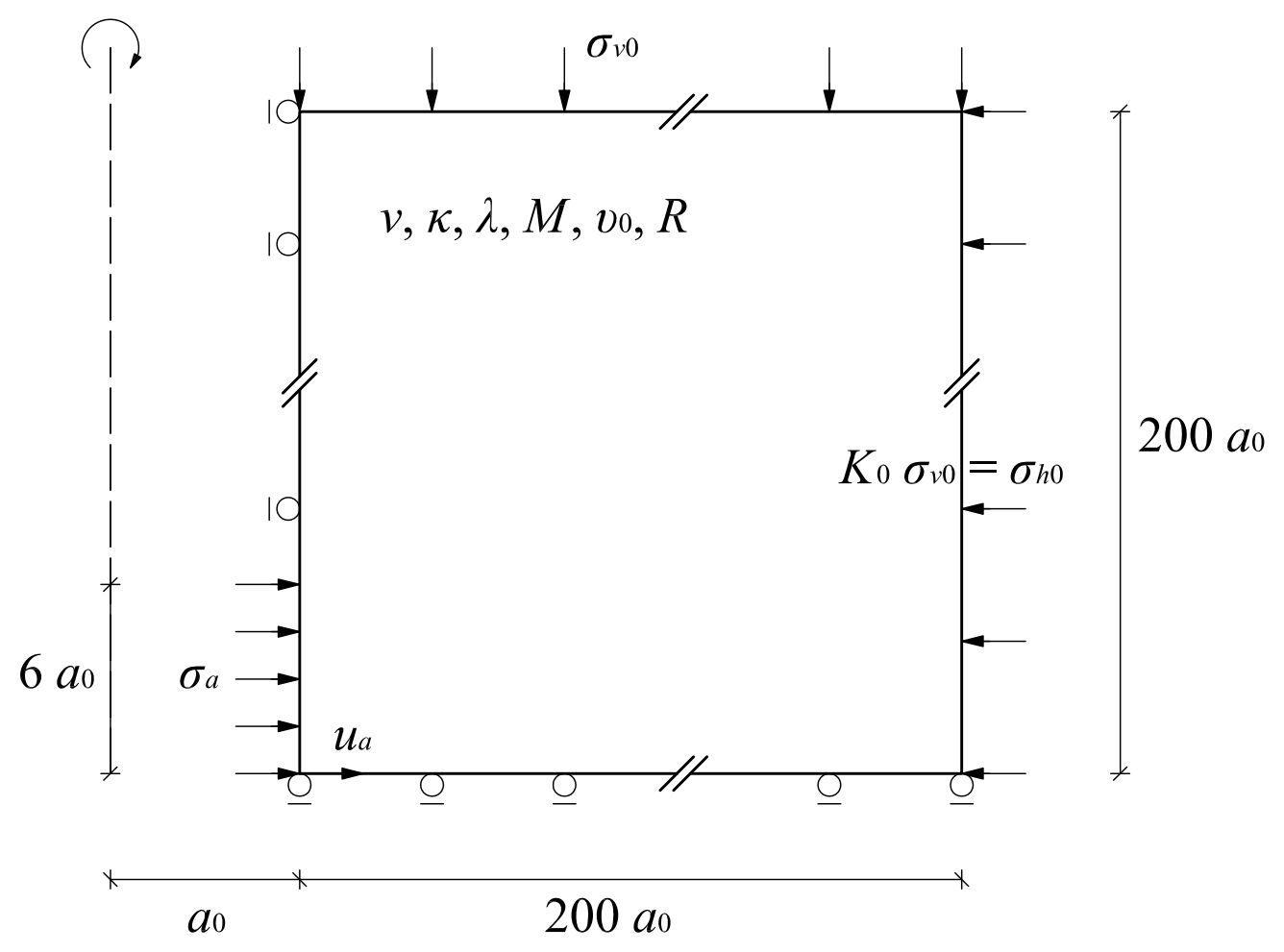




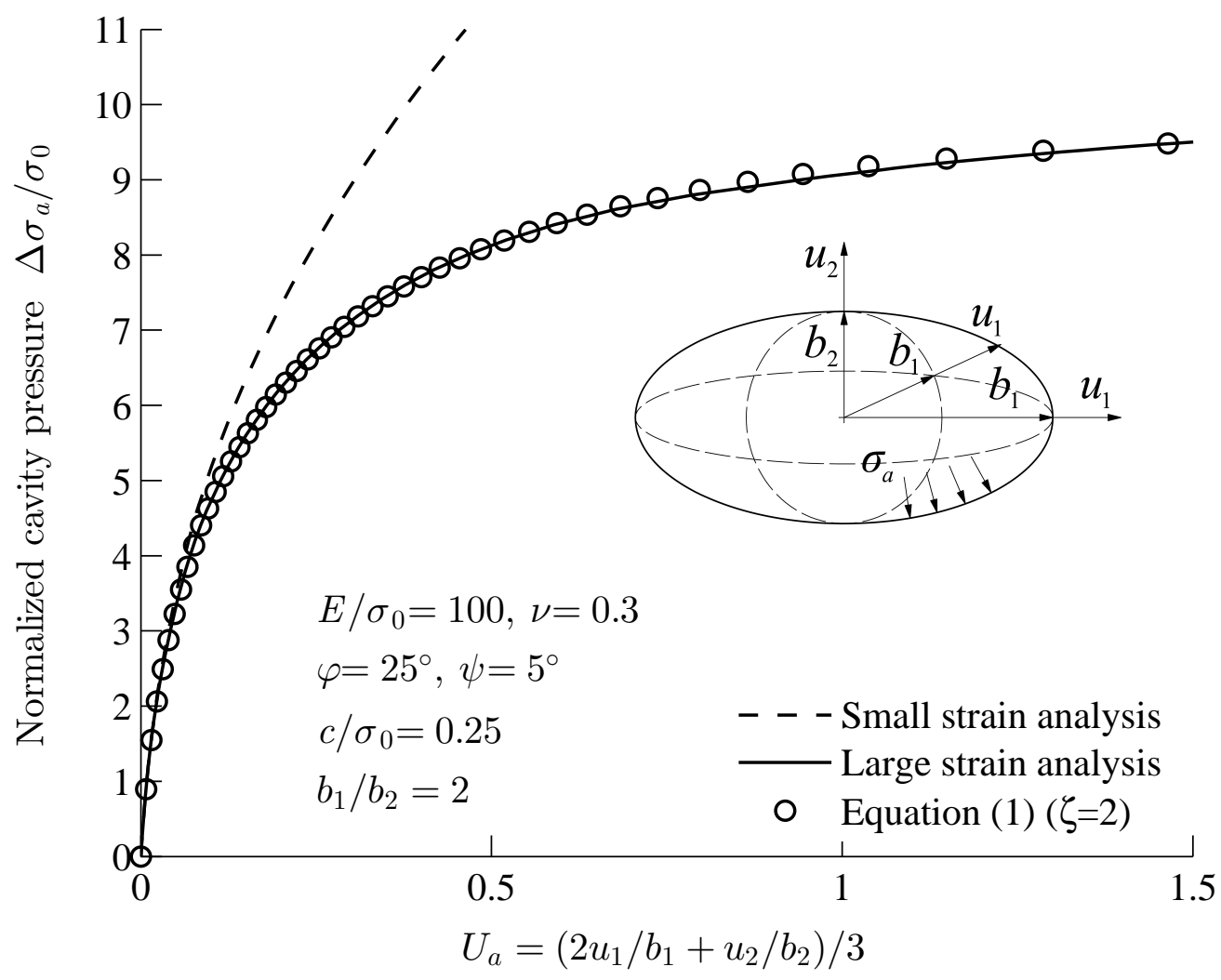




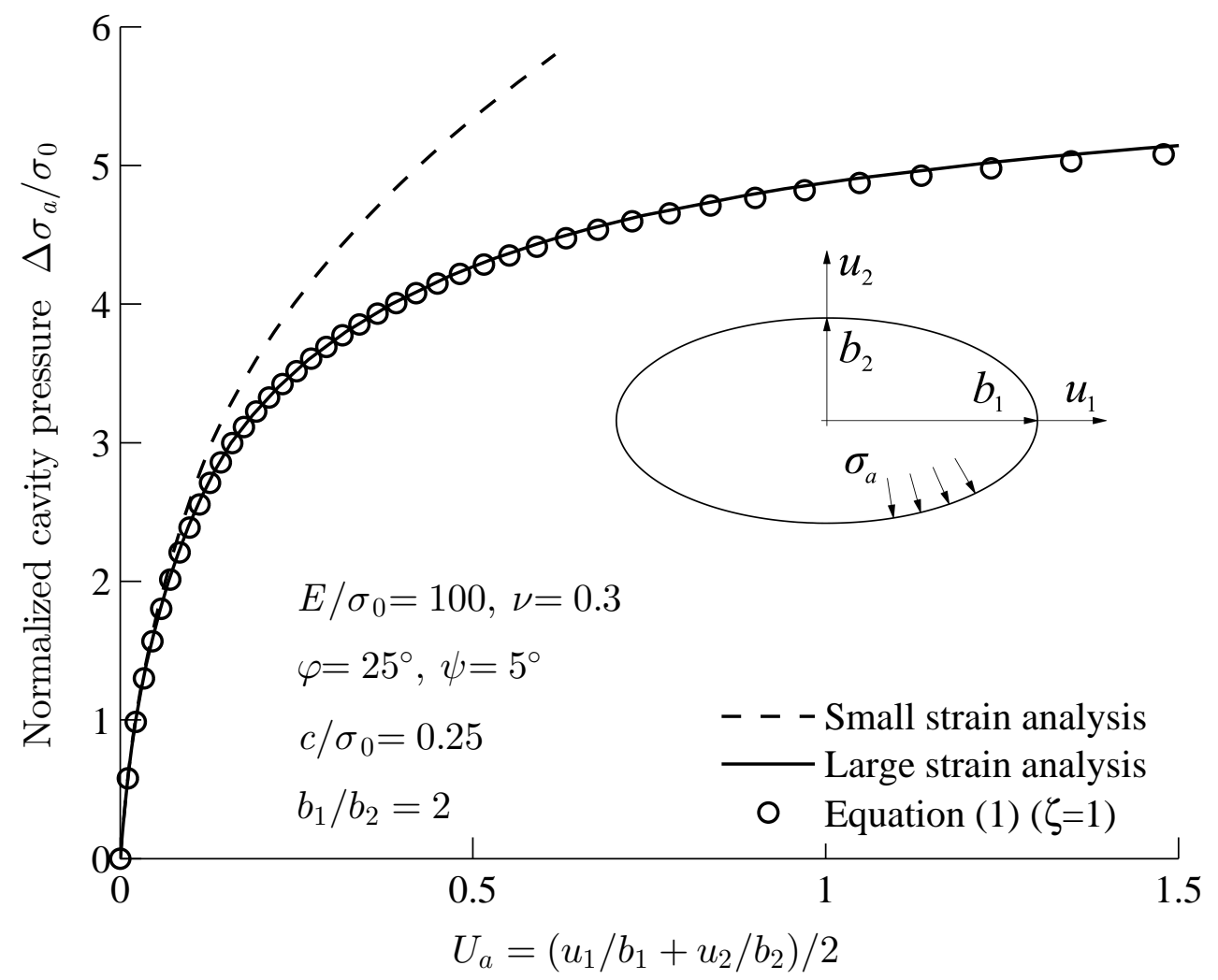




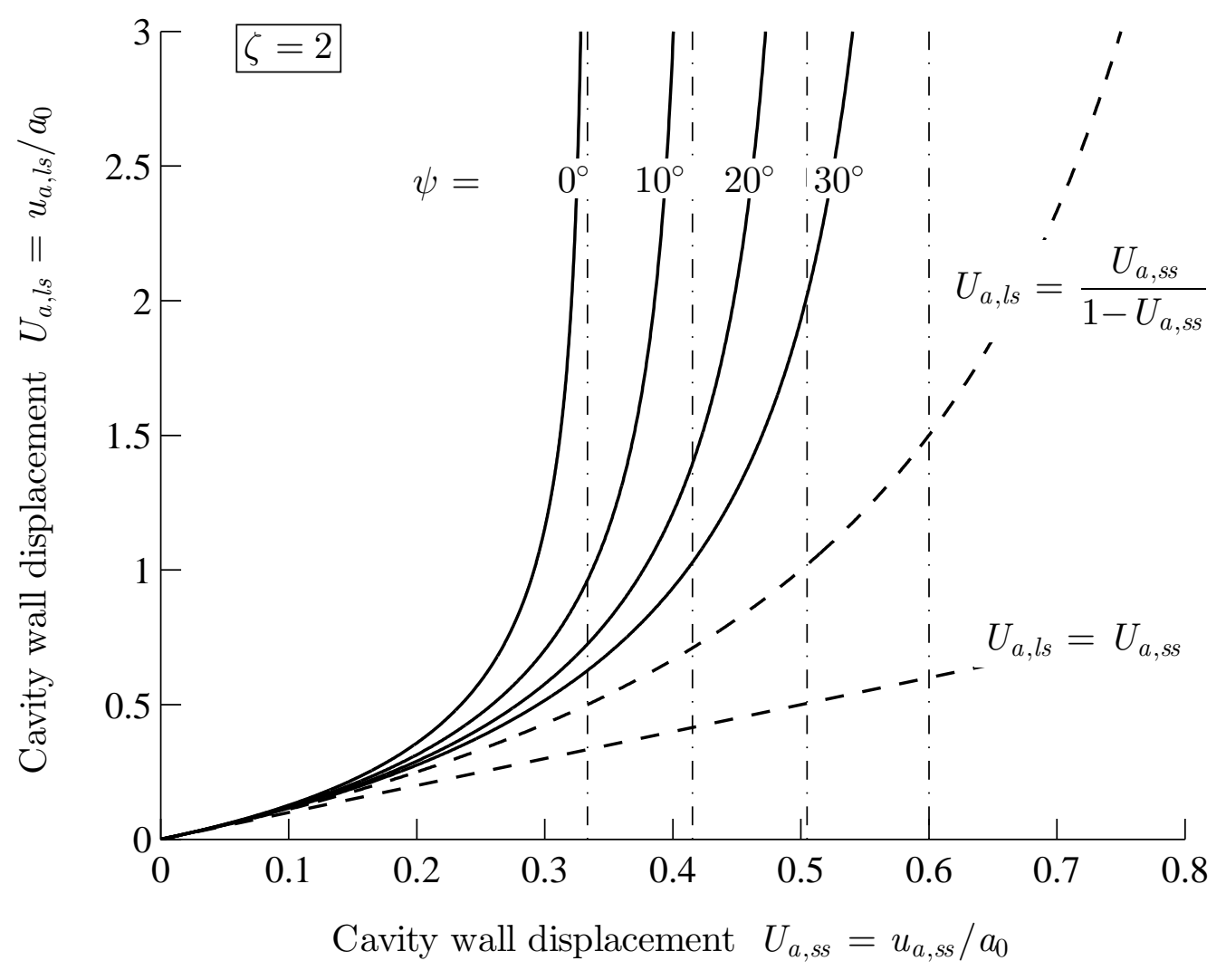




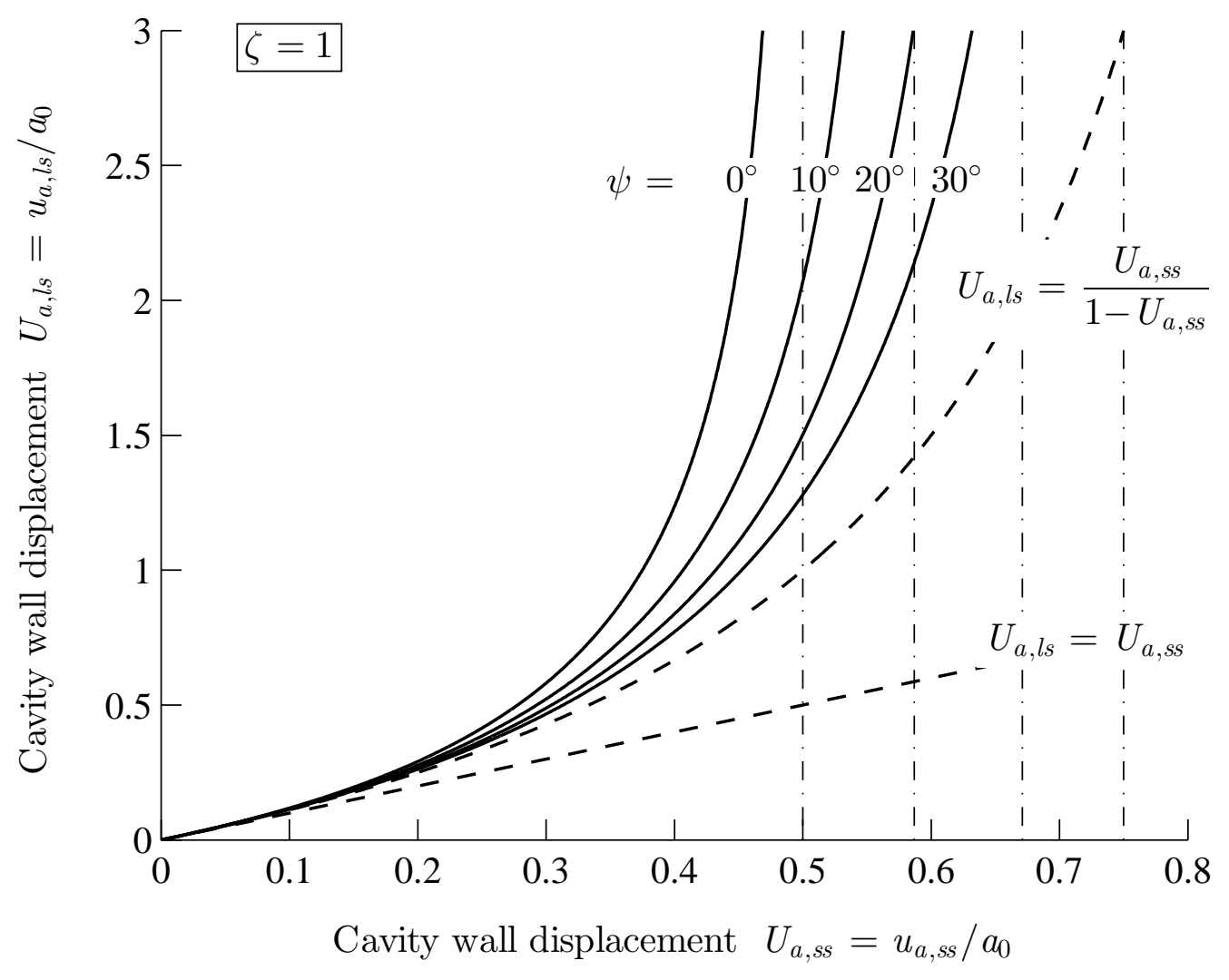



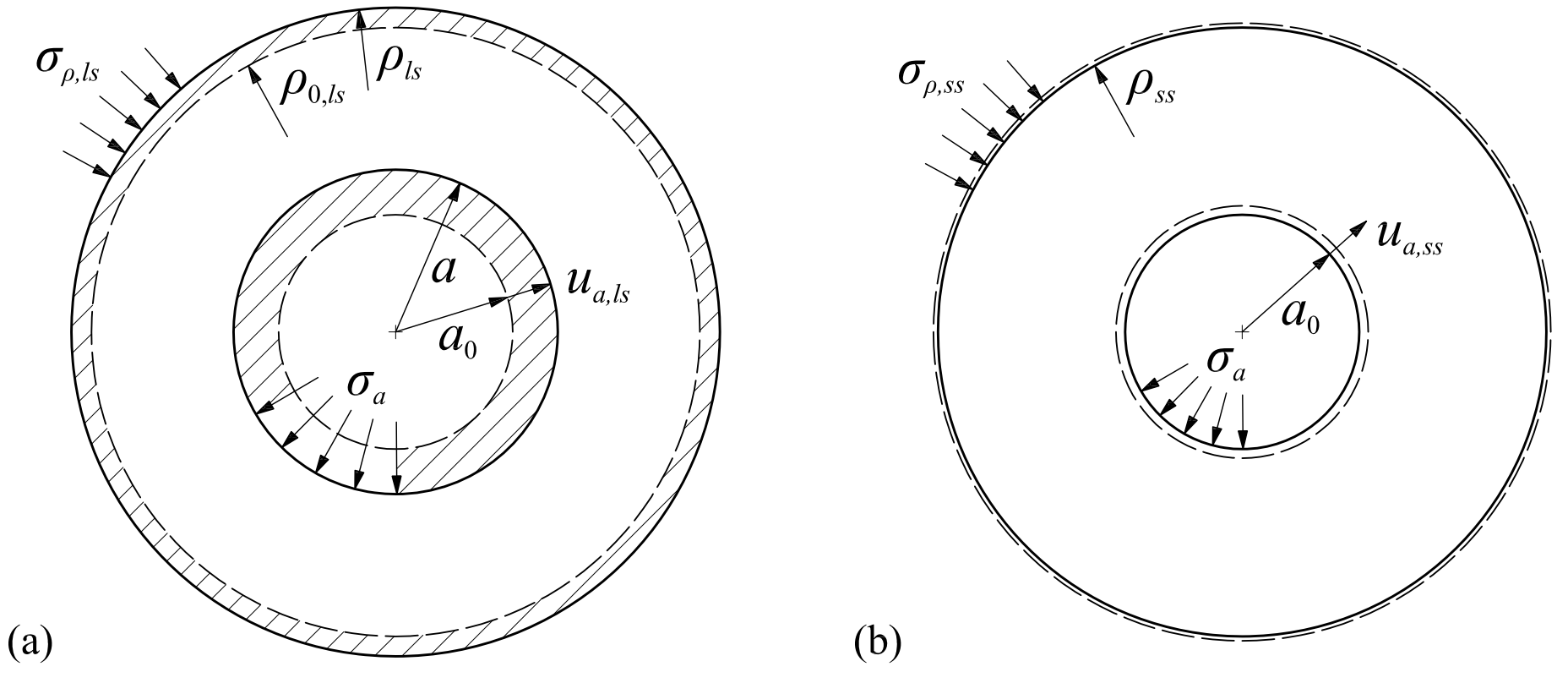


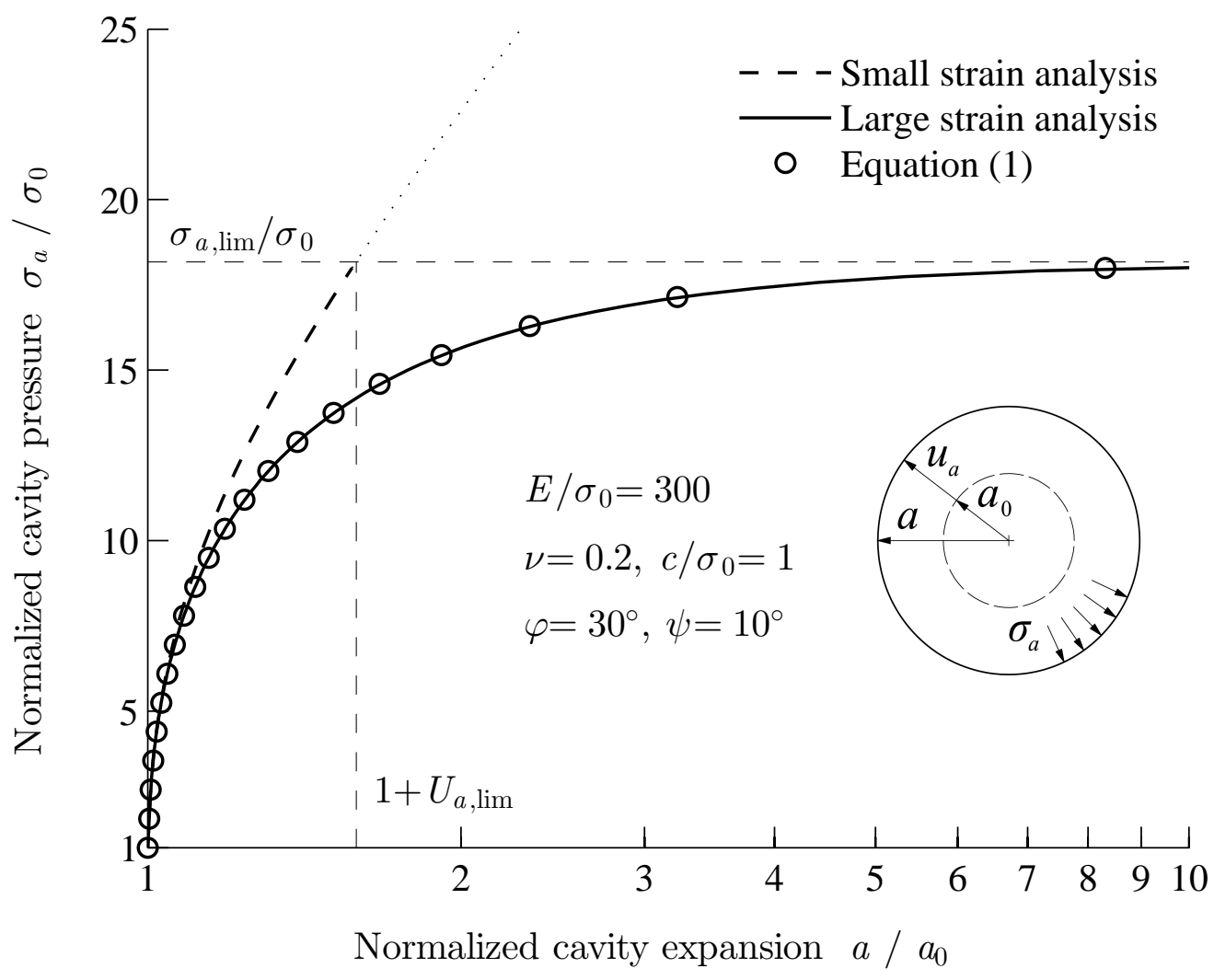

